\title{
Investigating the Effects of COVID-19 Pandemic on Stress, Anxiety, Depression, Self-care Behaviors, and the Quality of Life of Women at Risk of Preterm Labor
}

\author{
Roghayeh Dargahi' ${ }^{1}$, Zahra Bahrami-As| ${ }^{2}$, Abbasali Dorosti ${ }^{3}$, Fatemeh Mallah ${ }^{4 *}$
}

\begin{abstract}
Objectives: The coronavirus disease 2019 (COVID-19) pandemic increased the severity of the psychological problems of pregnant women, which can have severe consequences. The current study aimed to investigate the effects of COVID-19 on the stress, anxiety, depression, self-care behaviors, and the quality of the life of women at the risk of preterm labor.

Materials and Methods: The current descriptive-analytical study was conducted from March 20, 2020 to June 21, 2020. In total, 88 women who were at risk of preterm labor in Tabriz (Iran) participated in this study. The association between the Depression, Anxiety and Stress Scale-21 Items (DASS-21) questionnaire, Hart's self-care behaviors during pregnancy, and SF-36 (for the quality of life) were investigated using Pearson and Spearman correlations in SPSS 20 at $P<0.05$.

Results: Based on the results of the Pearson correlation test, stress and self-care scores $(P=0.003, \mathrm{r}=-482)$, self-care and depression scores $(P=0.006, \mathrm{r}=-396)$, as well as anxiety and self-care scores $(P=0.001, \mathrm{r}=-511)$ had significant inverse linear correlations. According to the regression model, only the stress variable had a significant association with self-care $(P=0.039)$. There was a positive and significant $(P<0.05)$ correlation between self-care behaviors and three dimensions of marital quality including physical function, mental function, and the feeling of pain and discomfort.

Conclusions: The results of the current study showed the significant inverse association between self-care and depression and stress and anxiety during pregnancy in women at risk of preterm labor during the COVID-19 pandemic. Low quality self-care behaviors also led to a decrease in the quality of life of these women.

Keywords: Stress, Depression, Self-care, COVID19, Preterm labor
\end{abstract}

\section{Introduction}

Stress during pregnancy causes adverse effects for the fetus, including low birth weight, preterm labor, reduced mental development at 18-24 months of age, extensive motor disorders, and other major complications (1-3). On the other hand, anxiety is one of the most common symptoms in pregnant women and is the main cause of labor pain. Babies born to stressed and anxious mothers are at an increased risk of restlessness and underweight $(4,5)$. Depression is another common disorder during pregnancy. Pregnancy depression is an important issue that affects approximately one-fifth of mothers and can lead to ignoring self-care and prenatal care, poor nutrition, smoking, self-medication, low birth weight, and preterm delivery (6-8).

Undoubtedly, mental illnesses such as stress and depression significantly increase the risk of preterm labor. According to the literature, there is a strong and negative association between the aforementioned disorders and self-care, and the psychological pressures of this period would be managed and the side effects of these psychological problems will be reduced if pregnant women can appropriately perform the self-care process (9-11).

The psychological aspects of the corona pandemic cause various stress and mental illness for pregnant women (12-14) and this pressure can decrease the quality of self-care programs while increasing the risk of preterm labor, affecting the quality of life and thus the maternal and infant health, as well as health care expenditures. Therefore, the current study sought to investigate the effects of COVID-19 on the stress, anxiety, depression, and self-care behaviors in predicting the quality of life of women at the risk of preterm delivery.

\section{Materials and Methods}

Study Design

The current descriptive-analytical study was performed from March 20, 2020 to June 21, 2020. In general, 88 women at the risk of preterm labor in Tabriz (Iran)

Received 9 June 2020, Accepted 24 September 2020, Available online 9 November 2020

'Department of Obstetrics and Gynecology, School of Medicine and Allied Medical Sciences, Ardabil University of Medical Sciences, Ardabil, Iran ${ }^{2}$ Anatomy and Biology Department, Medicine Faculty, Shahid Beheshti University of Medical Science, Tehran, Iran. ${ }^{3}$ Department of Anesthesiology, Medicine Faculty, Tabriz University of Medical Sciences, Tabriz, Iran. ${ }^{4}$ Obstetrics and Gynecology Department, Medicine Faculty, Tabriz University of Medical Science, Tabriz, Ira.

*Corresponding Author: Fatemeh Mallah, Te1: +984135539161, Email: Fatima.m815@yahoo.com 
Key Messages

- Stress reduces self-care behaviors in pregnant women with preterm labor.

- A reduction in self-care behaviors decreases the quality of life of pregnant women with preterm labor.

- During the corona pandemic (a stressful crisis for pregnant women), self-care behaviors reduce, leading to a severe decline in the quality of life.

participated in the current study. The minimum sample size was calculated using similar research (9). The results of the preliminary study and the sample size estimation formula were used for the correlation coefficient with a $95 \%$ confidence level, and $80 \%$ test. The study population included all pregnant women at the risk of preterm delivery who visited health centers affiliated to Tabriz University of Medical Sciences in Tabriz. Participants were selected using the random sampling technique so that a list of all pregnant women visiting health centers was prepared, and then they were selected randomly. The inclusion criteria were living in Tabriz, being aged at least 18 years old, showing a willingness to participate (both the women herself and her husband), having a gestational age (based on accurate and reliable last menstrual period provided through sonography during the first trimester), and obtaining a score of 10 or higher using the preterm labor questionnaires. On the other hand, the exclusion criteria were unwillingness to participate in the study, speech and hearing impairment or accent problems that prevented communication with the researcher, and the lack of completely filling the questionnaires.

\section{Data Collection Tools}

Holbrooke Early Childhood Screening Questionnaire, forms on demographic information of pregnant women and midwifery information, Hart Pregnancy Care Measures Questionnaire, and the Depression, Anxiety and Stress Scale-21 (DASS-21) Questionnaire were used to collect information. The Holbrooke questionnaire consists of three sections including the main (13 questions), secondary (14 questions), and background (12 questions) risk factors. Each question in the first section has 10 points while it has 5 and 2.5 points in the second and third parts, respectively. A score higher than 10 points indicates a high risk of preterm labor. However, a score of less than 10 represents a low risk of preterm labor. Women with a minimum score of 10 were included in this study.

Forms on the demographic information of pregnant women and midwifery information contain two parts (10 questions on demographic information and 20 questions on midwifery information). Hart's questionnaire is based on Orem's self-care items and pregnancy care guidelines developed by Hart. This questionnaire comprises 41 questions on a 5-point Likert-type scale (never, rarely, sometimes, often, and always). A higher score indicates better self-care (9).

The DASS-21 questionnaire helps in diagnosing and screening the signs of anxiety, depression, and stress over the past week. Each of its questions contains 4 options ranging from "never" to "always". A score of zero is equivalent to "Never" and a score of one is for "quantitative" options. In addition, the scores of two and three represent "Sometimes" and "always", respectively. The final score of each of the subscales should be doubled considering that this questionnaire is a short form that consists of 42 questions (15). Forms on demographic information of pregnant women and midwifery information contain data on age, level of education, income level, number of previous pregnancies, number of labors, number of abortions, number of preterm births, number of live births, number of dead births, and gestational age.

The other part of the instrument included the SF-36 Quality of Life Questionnaire (16). This questionnaire examines the quality of life in 8 different dimensions, including a feeling of general health, physical function, role disorder due to physical health, role disorder due to mental health, social function, feeling pain and discomfort, feeling happy, and psychological function. In this questionnaire, getting a higher score indicates a better quality of life

\section{Steps}

The research team referred to health centers to select the participants after obtaining the approval of the Vicechancellor for the Research and the Ethics Committee and a letter of introduction from Tabriz University of Medical Sciences (TUMS), and permission from the authorities of the health centers. First, the objectives, method, and confidentiality of the information were explained to the potential participants and written consent was obtained from them upon their agreement. Then, the prenatal screening questionnaire was filled to investigate eligibility criteria. In other words, they were eligible to participate if they had a score higher than 10. Then, forms on demographic and midwifery information, Hart pregnancy self-care questionnaire, and DASS-21 questionnaire were filled by the participants.

\section{Statistical Analysis}

Data were analyzed using SPSS software, version 20. Descriptive statistics were used to describe the data, including frequency, mean, and standard deviation (SD). The Pearson correlation coefficient was used to investigate the association between stress, anxiety, and depression, and self-care. Further, Pearson and Spearman correlations were applied to evaluate the association between stress, anxiety, and depression with intervening variables. For all tests, the reliability coefficient was $95 \%$, and the $P$ value was less than 0.05 . 


\section{Results}

In total, 221 pregnant women were at risk of preterm labor in Tabriz. They were continuously being monitored by health centers. Using the random sampling technique, 88 of them were selected, all of whom continued the study to the end. The mean \pm SD age of the participants and the gestational age (weeks) were $24.12 \pm 03.18$ years and $26.15 \pm 0.75$ weeks, respectively. Most participants (40 or $45.45 \%$ ) had non-academic degrees, and the level of income was "adequate" for most (45 or $51.13 \%$ ) pregnant women (Table 1).

The mean \pm SD score was $09.15 \pm 1.15,09.00 \pm 0.55$, and $08.55 \pm 1.15$ for anxiety intensity, the severity of stress, and depression intensity, respectively. The levels of anxiety, stress, and depression indicated that most participants had high levels of stress, anxiety, and depression. Moreover, the scores of the quality of life (Total: $45.18 \pm 5.45$ ) dimensions demonstrated that the highest and lowest scores were related to physical health $(49.10 \pm 5.25)$ and self-care behaviors $(33.19 \pm 4.20)$, respectively (Table 2 ).

The mean \pm SD score of self-care was $109.15 \pm 10.45$. Based on the results of the Pearson correlation test, there was a reverse and significant linear correlation between stress and self-care scores $(P=0.003, r=-482)$, self-care score and depression $(P=0.006, \mathrm{r}=-396)$, and anxiety and self-care scores $(P=0.001, \mathrm{r}=-511)$.

According to the multiple regression, a significant association was found between anxiety, stress, and depression scores, and self-care $(P=0.019, \mathrm{r}=-401)$. Based on the regression model, only the stress variable had a significant association with self-care $(P=0.039)$, and anxiety and depression were not significantly associated with self-care in the presence of this variable (Table 3 ).

Table 1. Demographic Characteristics and Midwifery Information of Pregnant Women at the Risk of Preterm Delivery

\begin{tabular}{lc}
\hline Variable & Level \\
\hline Age, mean \pm SD & $24.12 \pm 03.18$ \\
Education, No. $(\%)$ & $6(06.81)$ \\
Illiterate & $20(22.72)$ \\
Literacy & $40(45.45)$ \\
Non-academic & \\
Income, No. (\%) & $14(15.90)$ \\
Less than enough & $45(51.13)$ \\
Enough & $29(23.95)$ \\
Too much & $2.10 \pm 0.15$ \\
Number of pregnancies, mean \pm SD & $0.85 \pm 0.15$ \\
Number of deliveries, mean \pm SD & $0.75 \pm 0.10$ \\
Number of abortions, mean \pm SD & $0.55 \pm 0.10$ \\
The number of preterm births, mean \pm SD & $1.01 \pm 0.10$ \\
The number of living sages, mean \pm SD & $0.2 \pm 0.10$ \\
Number of dead children, mean \pm SD & $00.00 \pm 0.20$ \\
The number of stillbirths, mean \pm SD & $26.15 \pm 0.75$ \\
Gestational age (wk), mean \pm SD &
\end{tabular}

SD: Standard deviation.
According to the results of the regression model, the scores of anxiety, stress, and depression were as follows:

Self-care score $=0.18($ Anxiety $)+0.02($ Depression $)-0.50$ (Stresses)

The stress and level of education had a significant linear association with anxiety, stress, and depression based on the results (Table 4) of multiple regression for the impacts of contextual and intervening variables (i.e., age, level of education, income level, number of previous pregnancies, number of labors, number of abortions, number of preterm births, number of live births, number of dead births, and gestational age).

The mean \pm SD quality of life score was $45.18 \pm 5.45$, indicating a low quality of life. There was a positive and significant $(P<0.05)$ correlation between selfcare behaviors and three dimensions of marital quality including physical function, mental function, and feeling of pain and discomfort. The study of the correlation

Table 2. Frequency of Pregnant Women at Risk for Preterm Birth in Terms of Stress, Anxiety, Depression, and the Quality of Life During the COVID-19 Pandemic

\begin{tabular}{|c|c|}
\hline Variable & Level \\
\hline \multicolumn{2}{|l|}{ Depression } \\
\hline Normal & $9(10.22 \%)$ \\
\hline Mild & $11(12.50 \%)$ \\
\hline Medium & $23(26.13 \%)$ \\
\hline Severe & $39(44.32 \%)$ \\
\hline Very intense & $6(06.81 \%)$ \\
\hline \multicolumn{2}{|l|}{ Anxiety } \\
\hline Normal & $12(13.63 \%)$ \\
\hline Mild & $10(11.36 \%)$ \\
\hline Medium & $18(20.45 \%)$ \\
\hline Severe & $38(43.18 \%)$ \\
\hline Very intense & $10(11.36 \%)$ \\
\hline \multicolumn{2}{|l|}{ Stress } \\
\hline Normal & Normal \\
\hline Mild & Mild \\
\hline Medium & Medium \\
\hline Severe & Severe \\
\hline Very intense & Very intense \\
\hline Quality of life (Mean \pm SD) & $45.18 \pm 5.45$ \\
\hline Self-care behaviors (Mean \pm SD) & $33.19 \pm 4.20$ \\
\hline Feeling of general health $($ Mean \pm SD) & $39.45 \pm 5.18$ \\
\hline Physical function (Mean \pm SD) & $40.12 \pm 5.51$ \\
\hline Physical health (Mean \pm SD) & $49.10 \pm 5.25$ \\
\hline Mental health (Mean \pm SD) & $51.81 \pm 5.43$ \\
\hline Social function (Mean \pm SD) & $52.12 \pm 5.79$ \\
\hline Social function (Mean \pm SD) & $46.40 \pm 5.25$ \\
\hline Feeling pain and discomfort (Mean \pm SD) & $36.56 \pm 4.49$ \\
\hline Feeling happy (Mean \pm SD) & $39.81 \pm 4.75$ \\
\hline Psychological function (Mean $\pm \mathrm{SD}$ ) & $42.21 \pm 5.44$ \\
\hline
\end{tabular}

COVID-19: Coronavirus disease 2019; SD: Standard deviation. 
Table 3. Results of Multiple Regression Coefficients for the Association Between Stress, Anxiety, and Depression Scores With Self-care During Pregnancy in Women at the Risk of Preterm Delivery During the COVID-19 Pandemic

\begin{tabular}{lcccc}
\hline Variable & $\boldsymbol{\beta}$ & SE & $\boldsymbol{T}$ Value & $\boldsymbol{P}$ Value \\
\hline Constant & 163.1 & 2.2 & 70.8 & 0.001 \\
Stresses & -0.50 & 0.20 & 2.0 & 0.039 \\
Anxiety & 0.18 & 0.15 & 0.65 & 0.209 \\
Depression & 0.02 & 0.25 & 0.1 & 0.523 \\
\hline
\end{tabular}

COVID-19: Coronavirus disease 2019; SE, standard error.

Table 4. Results of Multiple Regression Coefficients for Investigating the Impacts of Contextual and Intervention Variables on the Association Between Stress, Anxiety, and Depression Scores and Self-care During Pregnancy in Women at the Risk of Preterm Labor During the COVID-19 Pandemic

\begin{tabular}{lcccc}
\hline Variable & $\boldsymbol{\beta}$ & SE & $\boldsymbol{T}$ Value & $\boldsymbol{P}$ Value \\
\hline Constant & 209.6 & 55.1 & 4.1 & 0.000 \\
Stresses & -0.43 & 0.19 & -1.6 & 0.035 \\
Anxiety & 0.15 & 0.23 & 0.33 & 0.559 \\
Depression & 0.03 & 0.36 & 0.71 & 0.801 \\
Age & -0.05 & 0.35 & -0.10 & 0.496 \\
Education & 4.21 & 1.49 & 2.30 & 0.019 \\
Income & -1.45 & 3.45 & -0.35 & 0.498 \\
Number of pregnancies & -8.78 & 15.75 & 0.80 & 0.639 \\
Number of deliveries & 10.10 & 17.90 & 0.49 & 0.603 \\
Number of abortions & 12.45 & 3.30 & -1.43 & 0.193 \\
The number of preterm births & -3.63 & 25.25 & -0.13 & 0.659 \\
The number of living sages & 1.73 & 25.53 & -0.12 & 0.759 \\
Number of dead children & 1.12 & 20.75 & 0.03 & 0.714 \\
The number of stillbirths & 1.40 & 20.19 & 0.19 & 0.914 \\
Gestational age (wk) & -2.43 & 1.29 & -1.2 & 0.211 \\
\hline
\end{tabular}

COVID-19: Coronavirus disease 2019; SE, standard error.

between the general scores of the quality of life and self-care behaviors also revealed that there is a positive and significant correlation between these two variables (Table 5).

\section{Discussion}

The current study aimed to evaluate the effects of the COVID-19 pandemic on stress, anxiety, depression, and self-care behaviors of women at the risk of preterm labor. According to the results, stress, anxiety, and depression had a significant and negative association with self-care. In other words, those with high levels of depression, stress, and anxiety had lower levels of self-care. The results of the present study are consistent with those of other similar studies (9,17-19) although there are differences. For example, the obtained scores for stress, anxiety, and depression are higher in the current study, and self-care scores are lower, which can be attributed to the concerns related to the COVID-19 pandemic. In other words, the COVID-19 pandemic increased the levels of stress, anxiety, and depression in pregnant women at risk for preterm labor while decreasing the self-care score more relative to previous studies.

The results of the present study showed a positive and significant correlation between the quality of life scores and self-care behaviors in women with preterm delivery in the corona pandemic period. On the other hand, it was found that the quality of life score is low, which predicts a low level of self-care behaviors in pregnant women with preterm delivery in the corona pandemic period. The results of our study are in line with the findings of a similar study (20). Given that the corona pandemic is a stressful period for pregnant women, those who are diagnosed with preterm labor due to the type of delivery are exposed to high levels of stress that can worsen, along with the critical situation.

Depression is characterized by a bad mood, decreased energy and interest, a sense of guilt, difficulty in concentrating, anorexia, suicidal thoughts, insomnia or drowsiness, significant weight loss, and dysfunction. Pregnancy depression may lead to inadequate nutrition, smoking, self-medication, lack of care during pregnancy, and the lack of self-care. It seems that self-care can increase the likelihood of following treatment methods while reducing the physical and psychological symptoms of diseases such as depression. Pregnant women who are depressed and have higher levels of stress have lower performance compared to non-depressed women. This lower performance can negatively affect self-care behaviors during pregnancy $(21,22)$. Hence, high stress

Table 5. Pearson Correlation Coefficient Matrix Between the Structures of the Theory of Self-care Behaviors and the Quality of Life in the Subjects

\begin{tabular}{|c|c|c|c|c|c|c|c|c|c|c|c|}
\hline & & 1 & 2 & 3 & 4 & 5 & 6 & 7 & 8 & 9 & 10 \\
\hline 1. & Self-care behaviors & 1 & & & & & & & & & \\
\hline 2. & Feeling of general health & $0.261^{* *}$ & 1 & & & & & & & & \\
\hline 3. & Physical function & -0.59 & $-0.311^{* *}$ & 1 & & & & & & & \\
\hline 4. & Physical health & -0.51 & $-0.281^{* *}$ & $0.600^{* *}$ & 1 & & & & & & \\
\hline 5. & Mental health & 0.121 & $0.401^{* *}$ & $-0.401^{* *}$ & $-0.395^{* *}$ & 1 & & & & & \\
\hline 6. & Social function & $0.169^{*}$ & $0.140^{*}$ & $-0.249^{* *}$ & $-0.300^{* *}$ & $0.500^{* *}$ & 1 & & & & \\
\hline 7. & Social function & 0.074 & $0.340^{* *}$ & $-0.371^{* *}$ & $-0.351^{* *}$ & $0.388^{* *}$ & $0.265^{* *}$ & 1 & & & \\
\hline 8. & Feeling pain and discomfort & $0.200^{* *}$ & $0.450^{* *}$ & $-0.451^{* *}$ & $-0.301^{* *}$ & $0.402^{* *}$ & $0.371^{* *}$ & $0.477^{* *}$ & 1 & & \\
\hline 9. & Feeling happy & 0.091 & $0.302^{* *}$ & $-0.385^{* *}$ & $-0.349^{* *}$ & $0.599^{* *}$ & $0.500^{* *}$ & $0.281^{* *}$ & $0.481^{* *}$ & 1 & \\
\hline 10. & Psychological function & $0.193^{* *}$ & $0.444^{* *}$ & $0.16^{3^{*}}$ & $0.259^{* *}$ & $0.498^{* *}$ & $0.444^{* *}$ & $0.443^{* *}$ & $0.520^{* *}$ & $0.473^{* *}$ & 1 \\
\hline
\end{tabular}

Note. *: It is significant at $P<0.05 ;{ }^{* *}$ : It is significant at $P<0.01$; The applied test: Pearson correlation. 
reduces the self-care behaviors of pregnant women at risk for preterm labor. The increased stress has much more adverse effects on self-care in stressful conditions such as the COVID-19 pandemic.

During pregnancy, maternal stress can cause consequences such as premature birth, low birth weight, and the risk of developing high blood pressure during pregnancy. Some researchers reported stress as an essential part of the pregnancy which has a crucial role in increasing the rate of biochemical changes in the mother's body. The researchers further reported a close association between maternal stress during pregnancy and a series of negative birth outcomes. Considering that pregnancy is a type of stress, its association with depression is natural. According to the literature, $50-80 \%$ of depressed people, experience some levels of stress until six months before the diagnosis of depression compared to 20-30\% of non-depressed people $(23,24)$. Meanwhile, some of the participants of the current study had problems such as stillbirth, miscarriage, or infertility that increase the likelihood of depression. Therefore, in situations that enhance the stress level (e.g., the COVID-19 pandemic), it is necessary to identify factors that increase the severity of stress so that programs can be arranged to address such factors.

Another important finding of the present study was the significant association between stress and the education level and self-care education in women at the risk of preterm labor during the COVID-19 pandemic. Similar research (25) also reported such an association. However, regarding the conditions and psychological atmosphere prevailing in the community during the COVID-19 pandemic, these associations were stronger compared to other studies (26-31). One possible reason for this finding is that the majority of illiterate women have a weak or mediocre economic status. Meanwhile, the important role of higher awareness and knowledge of educated people as a protective factor cannot be ignored, and it is quite usual that illiterate people have less knowledge about selfcare methods during the COVID-19 pandemic, which decreases the quality of self-care for pregnant women who are at the risk of preterm labor.

\section{Conclusions}

The results of the current study showed the significant inverse association between self-care and depression and stress and anxiety during pregnancy in women at the risk of preterm labor during the COVID-19 pandemic. Finally, low quality in self-care behaviors led to a decrease in the quality of life of these women.

\section{Limitations of the Study}

One of the limitations of the current study is that the researcher was not presented at the health centers to observe social distancing at the time of filling the questionnaires. Therefore, participants may have not properly understood the questions, and there was no one to provide extra information. Thus, the cross-sectional nature of the current study is another main limitation.

\section{Suggestions for Future Studies}

It is recommended to conduct studies with a longterm horizon and a larger sample size, as well as with the participation of women whose relatives or family members have/had COVID-19. The practical implication of the current study is the necessity of the psychological screening of pregnant women during the COVID-19 pandemic to perform psychological or therapeutic measures, if necessary.

\section{Authors' Contribution}

RD: Study design and intervention implementation; ZBA and AD: Intervention implementation and data collection; FM: Manuscript preparation and article submission.

\section{Conflict of Interests}

Authors declare that they have no conflict of interests.

\section{Ethical Issues}

The authors attempted to fully observe the principles of ethics in human research, including obtaining an ethical code from TUMS, a license from the Health Department of TUMS, and written consent from pregnant women and their husbands, as well as explaining the objectives of the study and observing social distancing in health centers while not receiving fees from the participants. The research project was approved by the Ethics Committee of Tabriz University of Medical Sciences (Ethics no. IR.TBZMED.REC. 1398.1306).

\section{Financial Support}

This study was granted by Tabriz University of Medical Sciences.

\section{Acknowledgments}

The researchers are grateful for the spiritual support provided by Tabriz University of Medical Sciences, as well as the patients who participated in the study.

\section{References}

1. Fakhari S, Bile Jani I, Atashkhouei S, Khanbabayi Gol M, Soliemanzadeh S. Comparing the effect of hypotension treatment due to spinal anesthesia with ephedrine or phenylephrine on arterial blood gases and neonatal Apgar score during cesarean delivery in obese mothers: randomized clinical trial. Iran J Obstet Gynecol Infertil. 2019;22(10):12-20. doi:10.22038/ ijogi.2019.14185

2. Ibrahim SM, Lobel M. Conceptualization, measurement, and effects of pregnancy-specific stress: review of research using the original and revised Prenatal Distress Questionnaire. J Behav Med. 2020;43(1):16-33. doi:10.1007/s10865-019-00068-7

3. Mahmoodpoor A, Abedini N, Parish M, Jannati A, Baradaran R. Efficacy of low dose interscalene brachial plexus block on post anesthesia recovery parameters after shoulder surgery. Pak J Med Sci. 2011;27:265-8.

4. Grigoriadis S, Graves L, Peer M, et al. Maternal anxiety during pregnancy and the association with adverse perinatal outcomes: systematic review and meta-analysis. J Clin Psychiatry. 2018;79(5):17r12011. doi:10.4088/JCP.17r12011

5. Mobaraki-AsI N, Mirmazhari R, Dargahi R, Hadadi Z, Montazer M. Relationships among personality traits, anxiety, depression, hopelessness, and quality of life in patients with breast cancer. Iran J Breast Dis. 2019;12(3):60-71. doi:10.30699/acadpub. ijbd.12.3.60

6. Aghamohamadi D, Khanbabayi Gol M. An investigation into 
the effects of magnesium sulfate on the complications of succinylcholine administration in nulliparous women undergoing elective cesarean section: a double-blind clinical trial. Int J Womens Health Reprod Sci. 2019;7(4):520-525. doi:10.15296/ ijwhr.2019.86

7. Gentile S. Untreated depression during pregnancy: short- and long-term effects in offspring. A systematic review. Neuroscience. 2017;342:154-166. doi:10.1016/j.neuroscience.2015.09.001

8. Alizadeh Sabeg P, Mehrabi E, Nourizadeh R, Poursharifi H, Mousavi S. The effect of counseling on breast cancer awareness in rural Iranian women: a randomized controlled clinical trial. J Cancer Educ. 2019;34(6):1083-1091. doi:10.1007/s13187-0181411-z

9. Rezaeian SM, Abedian Z, Latifnejad Roudsari R, Mazloom SR, Dadgar $S$. The relationship of prenatal self-care behaviors with stress, anxiety and depression in women at risk of preterm delivery. Iran J Obstet Gynecol Infertil. 2017;20(3):68-76. doi:10.22038/ ijogi.2017.8874

10. Seyed Hashemi E, Dargahi R, Entezari M, Hosseini As SSI. Expression of miR200a as a biomarker in women with preterm delivery. Iran J Obstet Gynecol Infertil. 2019;22(7):39-46. doi:10.22038/ijogi.2019.13816

11. Nikkhesal N, Nourizadeh R, Dastgiri S, Mehrabi E. The factors affecting women's decision about unplanned pregnancy: a hierarchical modeling strategy. Int J Womens Health Reprod Sci. 2018;6(4):483-490. doi:10.15296/ijwhr.2018.80

12. Kurdo冈lu M, Khaki A. What is the current state of knowledge about the novel coronavirus infection during pregnancy? Int J Womens Health Reprod Sci. 2020;8(2):110-111. doi:10.15296/ ijwhr.2020.17

13. Haghdoost SM, Khanbabayi Gol M. The necessity of paying more attention to the neurological and psychological problems caused by COVID-19 pandemic during pregnancy. Int J Womens Health Reprod Sci. 2020;8(3):243-244. doi:10.15296/ijwhr.2020.40

14. Ataei-Almanghadim K, Farshbaf-Khalili A, Ostadrahimi AR, Shaseb E, Mirghafourvand M. The effect of oral capsule of curcumin and vitamin $\mathrm{E}$ on the hot flashes and anxiety in postmenopausal women: a triple blind randomised controlled trial. Complement Ther Med. 2020;48:102267. doi:10.1016/j.ctim.2019.102267

15. Nagandla K, Nalliah S, Yin L, et al. Prevalence and associated risk factors of depression, anxiety and stress in pregnancy. Int J Reprod Contracept Obstet Gynecol. 2016;5(7):2380-2388. doi:10.18203/2320-1770.ijrcog20162132

16. Gu M, Cheng Q, Wang X, et al. The impact of SLE on health-related quality of life assessed with SF-36: a systemic review and meta-analysis. Lupus. 2019;28(3):371-382. doi:10.1177/0961203319828519

17. Mochache K, Mathai M, Gachuno O, Vander Stoep A, Kumar M. Depression during pregnancy and preterm delivery: a prospective cohort study among women attending antenatal clinic at Pumwani Maternity Hospital. Ann Gen Psychiatry. 2018;17:31. doi:10.1186/ s12991-018-0202-6

18. Mahmoodpoor A, Ali-Asgharzadeh A, Parish M, Amir-Aslanzadeh $Z$, Abedini N. A comparative study of efficacy of intensive insulin therapy versus conventional method on mortality and morbidity of critically ill patients. Pak J Med Sci. 2011;27(3):496-499.

19. Parish M, Mahmoodpoor A, Sanaie S. Validity of fasting blood sugar on day of surgery compared with the preinduction blood glucose level in type II diabetic patients. Pak J Med Sci. 2007;23(2):202205.

20. Rezaeean SM, Abedian Z, Latifnejad Roudsari R, Mazloom SR Dadgar S. Application of Orem's theory for promotion of selfcare behaviors of pregnant women at risk for preterm delivery: a clinical trial. Iran J Obstet Gynecol Infertil. 2017;20(2):68-77. doi:10.22038/ijogi.2017.8717

21. Howdeshell KL, Ornoy A. Depression and its treatment during pregnancy: overview and highlights. Birth Defects Res. 2017;109(12):877-878. doi:10.1002/bdr2.1080

22. Mitchell J, Goodman J. Comparative effects of antidepressant medications and untreated major depression on pregnancy outcomes: a systematic review. Arch Womens Ment Health. 2018;21(5):505-516. doi:10.1007/s00737-018-0844-z

23. Alaem F, Jalali A, Almasi A, Abdi A, Khalili M. Investigating the effect of group counseling on family stress and anxiety of primiparous mothers during delivery. Biopsychosoc Med. 2019;13:7. doi:10.1186/s13030-019-0148-1

24. Dennis-Tiwary TA, Denefrio S, Gelber S. Salutary effects of an attention bias modification mobile application on biobehavioral measures of stress and anxiety during pregnancy. Biol Psychol. 2017;127:148-156. doi:10.1016/j.biopsycho.2017.05.003

25. Azh N, Ghasemi M, Khani M, Mafi M, Ranjkesh F. Relationship between maternal stress and pregnancy outcomes: a prospective study. Iran J Obstet Gynecol Infertil. 2019;22(5):27-36. doi:10.22038/ijogi.2019.13579

26. Hajian S, Mehrabi E, Simbar M, Houshyari M. Coping strategies and experiences in women with a primary breast cancer diagnosis. Asian Pacific journal of cancer prevention: APJCP. 2017;18(1):215. doi: 10.22034/APJCP.2017.18.1.215.

27. Nikkhesal N, Nourizadeh R, Dastgiri S, Mehrabi E. The factors affecting women's decision about unplanned pregnancy: A hierarchical modeling strategy. Int J Womens Health Reprod Sci. 2018;6(4):483-90. doi: 10.15296/ijwhr.2018.80.

28. Sabeg PA, Mehrabi E, Nourizadeh R, Poursharifi H, Mousavi S. The effect of counseling on breast cancer awareness in rural Iranian women: a randomized controlled clinical trial. J Cancer Educ. 2019;34(6):1083-1091. doi: 10.1007/s13187-018-1411-z.

29. Saboktakin L, Rafeey M, Kousha A, Moradi SM. Study on prevalence of Helicobacter pylori infection in adolescents with failure to thrive to compare with control group. Life Sci J. 2012;9:1425-1431

30. Saboktakin L, Barzegar M, Hagh Jo A, Emamalizadeh M. Study on serum copper and zinc level of children with epilepsy during long term therapy with anticonvulsants. Life Sci J. 2012;9(4):12501254.

31. Shayganmehr A, Dorosti AA, Saboktakin L, et al. Clinical Pediatric Screening for COVID-19. Iranian Journal of Pediatrics. 2021;31(1): e107780. doi: 10.5812/ijp.107780.

(c) 2022 The Author(s); This is an open-access article distributed under the terms of the Creative Commons Attribution License (http:// creativecommons.org/licenses/by/4.0), which permits unrestricted use, distribution, and reproduction in any medium, provided the original work is properly cited. 